\title{
An Anatomical Substrate for the Spatiotemporal Transformation
}

\author{
A. K. Moschovakis, ${ }^{2}$ T. Kitama, ${ }^{1}$ Y. Dalezios, ${ }^{2}$ J. Petit, ${ }^{1}$ A. M. Brandi, ${ }^{1}$ and A. A. Grantyn ${ }^{1}$ \\ ${ }^{1}$ Laboratoire de Physiologie de la Perception et de l'Action, Centre National de la Recherche Scientifique-College de \\ France, 75005 Paris, France and ${ }^{2}$ Division of Computational Neurosciences, Institute of Applied and Computational \\ Mathematics, Foundation for Research and Technology-Hellas, and Department of Basic Sciences, Faculty of Medicine, \\ University of Crete, GR-7110 Iraklion, Greece
}

\begin{abstract}
The purpose of the present experiments was to test the hypothesis that the metrics of saccades caused by the activation of distinct collicular sites depend on the strength of their projections onto the burst generators. This study of morphofunctional correlations was limited to the horizontal components of saccades. We evoked saccades by stimulation of the deeper layers of the superior colliculus (SC) in alert, head-fixed cats. We used standard stimulus trains of 350 msec duration, $200 \mathrm{~Hz}$ pulse rate, and intensity set at two times saccade threshold in all experiments. Evoked saccades were analyzed quantitatively to determine the amplitude of the horizontal component of their "characteristic vectors". This parameter is independent of eye position and was used as the physiological, saccade-related metric of the stimulation sites. Anatomical connections arising from these sites were visualized after anterograde transport of
\end{abstract}

biocytin injected through a micropipette adjoining the stimulation electrode. The stimulation and injection sites were, therefore, practically identical. We counted boutons deployed in regions of the paramedian pontine reticular formation reported to contain long-lead and medium-lead burst neurons of the horizontal burst generator. Regression analysis of the normalized bouton counts revealed a significant positive correlation with the size of the horizontal component of the characteristic vectors. This data supports a frequent modelling assumption that the spatiotemporal transformation in the saccadic system relies on the graded strength of anatomical projections of distinct SC sites onto the burst generators.

Key words: saccades; superior colliculus; burst generator; oculomotor system; orienting behavior; biocytin
The neural system that controls saccades is one of the best understood complex systems in the mammalian brain. Its principal cell classes have been described in terms of movement-related discharge patterns, topography, and connections (for review, see Moschovakis et al., 1996). As with other motor systems, it is characterized by transformations of signals as they pass from higher order supranuclear structures to motoneurons. One such transformation is called "spatiotemporal" because it links portions of the saccadic system that use a space code to those that use a temporal code to specify the same movement parameters. The saccadic burst generators are typical of structures that use a temporal code to specify movement amplitude. The size of the horizontal component of saccades is encoded by long-lead (LLB) and medium-lead (MLB) burst neurons of the paramedian pontine reticular formation (PPRF). Other LLB and MLB neurons located in the rostral mesencephalon encode the size of the vertical component of saccades. The saccadic burst generators receive their main input from the superior colliculus (SC). This structure uses a place code to specify the amplitude and direction of saccades in that it contains cells that emit high frequency bursts before saccades of particular direction and amplitude (Wurtz and Goldberg, 1972; Sparks and Mays, 1980). Cells of the rostral SC

\footnotetext{
Received June 25, 1998; revised Sept. 16, 1998; accepted Sept. 22, 1998.

This work was supported by Human Capital and Mobility Grant ERBCHRXCT940559. We gratefully acknowledge the comments of Dr. G. Delides and of an anonymous referee, the help of M. Pagomenou and G. G. Gregoriou with the analysis of the histological material, M. Chat and M. A. Thomas with the histochemistry, and S. Exinger with the photomicrographs.

Reprint requests should be addressed to Dr. Alexej Grantyn, Laboratoire de Physiologie de la Perception et de l'Action, College de France-Centre National de la Recherche Scientifique, 11 Place Marcelin Berthelot, 75005 Paris, France. Copyright (C) 1998 Society for Neuroscience 0270-6474/98/1810219-11\$05.00/0
}

discharge optimally for saccades having small horizontal components, whereas cells of the caudal SC prefer saccades with large horizontal components.

Early models of the spatiotemporal transformation assumed that the spatial concentration of cells projecting to the vertical and horizontal burst generators of the saccadic system varies systematically within the collicular map (McIlwain, 1982). In their pioneering effort to test this hypothesis, Edwards and Henkel (1978) described a rostrocaudal gradient of the number of tectal neurons projecting to the region of the abducens nucleus. According to this study, such tectoreticular cells become increasingly more numerous at successively more caudal levels of the feline SC (Edwards and Henkel, 1978). Compared to a rostral SC site, the excitation of a caudal SC site should then engage a larger number of SC neurons projecting to the horizontal saccade generator in the PPRF, and such a gradient would account for the spatiotemporal transformation in the horizontal plane. However, several studies failed to confirm the observations of Edwards and Henkel (Kawamura and Hashikawa, 1978; Stanton and Greene, 1981; Olivier et al., 1991). Alternatively, the spatiotemporal transformation could be based on the number of terminals that tectal efferent fibers deploy within the confines of regions housing the horizontal burst generator (Keller, 1980; Scudder, 1988; Moschovakis, 1994). To test this hypothesis, we combined a quantitative study of the anatomical projections to the PPRF, as revealed by circumscribed tracer injections into the SC, with a quantitative study of the metrics of saccades evoked by the electrical stimulation of the injection sites.

Parts of this paper have been presented in abstract form (Grantyn et al., 1997). 


\section{MATERIALS AND METHODS}

Experiments were performed on six adult cats weighing 3-4 kg. They were surgically prepared under pentobarbital anesthesia $(30 \mathrm{mg} / \mathrm{kg}$, i.p.) and sterile conditions. A search coil consisting of three loops of tefloninsulated stainless steel wire was wound under the insertions of the extraocular muscles. Stainless steel bolts were cemented onto the occipital and temporal bones for painless head fixation. After craniotomy, the dura mater was excised, and a cylindrical plastic chamber was placed over the cortical surface and cemented to the bone. The position of the chamber was determined stereotaxically so that vertically or obliquely oriented electrodes would have access to the superior colliculus. To prevent postoperative infections animals received intramuscular injections of a long-acting antibiotic (Extencilline). Four to seven days of postoperative recovery were allowed before starting the experiments. The animals were placed in a body-restraining cloth bag in front of a featureless screen, in a dimly illuminated room. Their heads were fixed to a stereotaxic frame by means of the implanted bolts. Drowsiness was prevented by alerting stimuli (noises, spots of light, morsels of food) presented at irregular intervals. To record the instantaneous horizontal and vertical position of the animals' eyes, their heads were centered within two magnetic fields with orthogonal directions and phase. The current induced in the eye coil was demodulated to obtain the vertical and horizontal components of instantaneous eye position with a resolution of $0.1^{\circ}$. Eye position was sampled at a rate of $500 \mathrm{~Hz}$ with a microcomputer running Spike2 software (Cambridge Electronics Design) and stored on disk for off-line analysis.

A double barrel micropipette assembly was lowered in the brain and advanced to the SC. The assembly was constructed by fixing together a graduated microsampling pipette $[1-5 \mu \mathrm{l}, 1.0 \mathrm{~mm}$ outer diameter (o.d.); Corning, Corning, NY; catalog \# 7099U] and a glass tube (1.5 mm o.d.) containing a tungsten wire. The tubes were heated inside the spiral of a vertical puller, twisted, and pulled to produce a double electrode with long taper. The glass was then broken off to obtain an injection channel with a diameter of $10-20 \mu \mathrm{m}$. The excess length of the tungsten wire was reduced by electrolysis to a sharp tip of $80-100 \mu \mathrm{m}$ in length, and the base of the tip was aligned with the orifice of the injection channel. The major diameter of the double pipette at the tip was $\sim 80 \mu \mathrm{m}$. Recording multiunit activity through the tungsten electrode allowed the detection of the collicular surface and a crude judgment of the directional tuning of visually responsive cells in the superficial tectal layers. When visual activity became weak, the tungsten electrode was switched from recording to stimulation to evoke saccades and to determine the depth of minimal threshold. We defined the threshold as the current intensity needed to evoke a saccade in about half of the trials. Electrical stimuli were delivered in trains of 70 cathodal pulses, $0.4 \mathrm{msec}$ in duration, and at an interval of $5 \mathrm{msec}$. These parameters were chosen from the literature (Guitton et al., 1980; Paré et al., 1994; Grantyn et al., 1996) to ensure that the amplitude of saccades evoked from each site would be maximal. Individual pulses were recorded as events with a time resolution of $10 \mu \mathrm{sec}$ and stored on disk for later analysis.

Because the amplitude of saccades electrically evoked from the SC has been shown to increase somewhat with stimulus intensity (Straschill and Rieger, 1973; Stein et al., 1976; Grantyn et al., 1996), we defined their size at current intensities equal to $2 \times \mathrm{T}(20-50 \mu \mathrm{A})$. The metrics of evoked saccades are also known to depend on the position of the eyes at the time of saccade onset (Straschill and Rieger, 1973; McIlwain, 1986, 1990; Grantyn et al., 1996). Therefore, even with the use of standard stimuli of equal relative intensities, the site-specific saccade metrics cannot be obtained by a simple averaging across a number of evoked saccades. To eliminate the effect of eye position, we used the method proposed by McIlwain $(1986,1988)$, which is based on the evaluation of the linear regression of horizontal (vertical) saccadic components, $\Delta \mathrm{H}$ $(\Delta \mathrm{V})$, versus the initial horizontal $\left(\mathrm{H}_{1}\right)$ or vertical $\left(\mathrm{V}_{1}\right)$ eye position. These expressions are of the form:

$$
\Delta \mathrm{H}=\beta_{\mathrm{H}}+\mathrm{a}_{\mathrm{H}} \mathrm{H}_{1} \text { and } \Delta \mathrm{V}=\beta_{\mathrm{V}}+\mathrm{a}_{\mathrm{V}} \mathrm{V}_{1} .
$$

Figure 1 provides an example from one of the animals of the present study (NB3). The slopes, $\mathrm{a}_{\mathrm{H}}$ and $\mathrm{a}_{\mathrm{V}}$, of these regression lines provide $\mathrm{a}$ measure of the position sensitivity of saccades. The constants $\beta_{\mathrm{H}}\left(13.5^{\circ}\right)$ and $\beta_{\mathrm{V}}\left(5.6^{\circ}\right)$ indicate the amount of eye displacement (horizontal and vertical) that would be obtained had the eyes started from primary position $\left(\mathrm{H}_{1}=0\right.$ and $\left.\mathrm{V}_{1}=0\right)$. The vector that corresponds to these values $\left(13.5,5.6^{\circ}\right)$ is defined as the "characteristic" vector of the saccades evoked from the collicular microzone that was electrically activated in animal NB3.

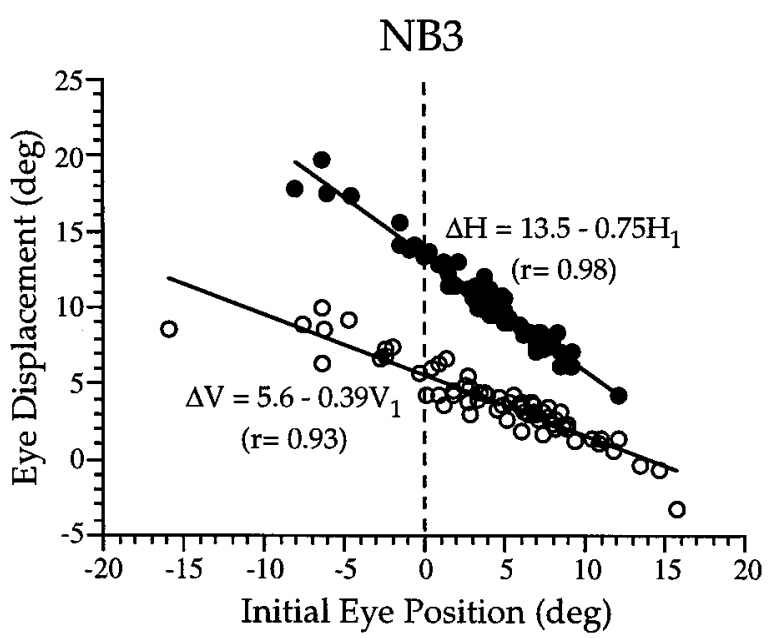

Figure 1. Plots of horizontal $(\Delta \mathrm{H})$ and vertical $(\Delta \mathrm{V})$ size of saccades (ordinate) elicited in response to the electrical stimulation $(2 \times \mathrm{T}, 70$ pulses) of the superior colliculus versus initial horizontal $\left(\mathrm{H}_{1}\right)$ and vertical $\left(\mathrm{V}_{1}\right)$ eye position (abscissa). Solid lines are the linear regression lines through the data (open circles, vertical; solid circles, horizontal) and obey the expressions displayed. The "characteristic vector" can be read off the points at which the linear regression lines intersect the vertical dashed line through zero initial eye position.

After collecting a sample of eye movement data, $0.8 \mu \mathrm{l}$ of biocytin ( $N$-biotinyl-L-lysine; Sigma, St. Louis, MO; 5\% solution in $100 \mathrm{~mm}$ Tris buffer, $\mathrm{pH}$ 7.4) was pressure injected in the SC through the second graduated barrel of the micropipette assembly. The injection of the solution was made slowly, over at least $30 \mathrm{~min}$, and the array was left in place for another $15 \mathrm{~min}$ before it was slowly retracted. Animals were allowed to survive for $60 \mathrm{hr}$ after the biocytin injections. They were then deeply anesthetized with pentobarbital $(60 \mathrm{mg} / \mathrm{kg}$, i.p. $)$, heparinized (5000 U, i.v.) through the femoral vein, and perfused transcardially with 11 of PBS, pH 7.4, followed by 21 of $4 \%$ paraformaldehyde dissolved in $100 \mathrm{~mm}$ phosphate buffer. The brain was removed, blocked in the plane of the electrode tracks, and stored in a $20 \%$ solution of sucrose in phosphate buffer. After equilibration, $75 \mu \mathrm{m}$ serial frontal sections were cut on a freezing microtome and reacted according to a modified version of the procedure used by Horikawa and Armstrong (1988). Briefly, sections were rinsed four times (20 min each) in a solution of PBS containing $0.1 \%$ Triton $\mathrm{X}-100$. Then, they were incubated overnight at room temperature in a 1:200 solution of avidin-biotin-peroxidase complex (ABC kit; Vector Laboratories, Burlingame, CA) in PBS containing $0.1 \%$ Triton $\mathrm{X}-100$. They were then rinsed three times $(10 \mathrm{~min}$ each $)$ in $0.05 \mathrm{M}$ Tris buffer, $\mathrm{pH}$ 7.4. The presence of bound HRP was revealed using a modified version of the heavy metal intensification of the DAB $\left(3^{\prime}-3^{\prime}\right.$-diaminobenzidine) reaction product (Adams, 1981). Briefly, sections were preincubated for $5-10 \mathrm{~min}$ in a $0.2 \%$ solution of nickel ammonium sulfate in Tris buffer, and then placed in a solution of Tris buffer containing $0.05 \%(\mathrm{w} / \mathrm{v}) \mathrm{DAB}$ and $0.2 \%(\mathrm{w} / \mathrm{v})$ nickel ammonium sulfate for $10 \mathrm{~min} . \mathrm{H}_{2} \mathrm{O}_{2}$ was added to this solution to a final concentration of $0.006 \%(\mathrm{v} / \mathrm{v})$, and the sections were incubated for an additional 5-20 min at room temperature. Reacted sections were rinsed three times in Tris buffer (10 min each), mounted on chromalum-gelatin-coated slides, and left overnight to dry. They were then cleared, lightly counterstained, dehydrated in a graded series of alcohols, and coverslipped.

The evaluation of morphological data was done with the help of a Zeiss Axioskope equipped with a drawing tube facing a monitor screen. The optical encoders of the microscope stage were connected to a Compaq 486 computer running reconstruction software (Neurolucida; MicroBrightField, Colchester, VT). The tracing of outer contours of the sections and of the outlines of selected nuclei was done using a $2.5 \times$ objective. The positions of retrogradely labeled cells and projection axons were entered at a higher magnification (200×). Subsequently, entire sections were systematically scanned to detect and plot the location of boutons. The scanning was done with a $40 \times$ objective. A $60 \times$ oil immersion objective was used to identify weakly stained boutons. The superimposition of sections and bouton counts within specified regions of the sections were obtained with the Neurolucida software. 
A

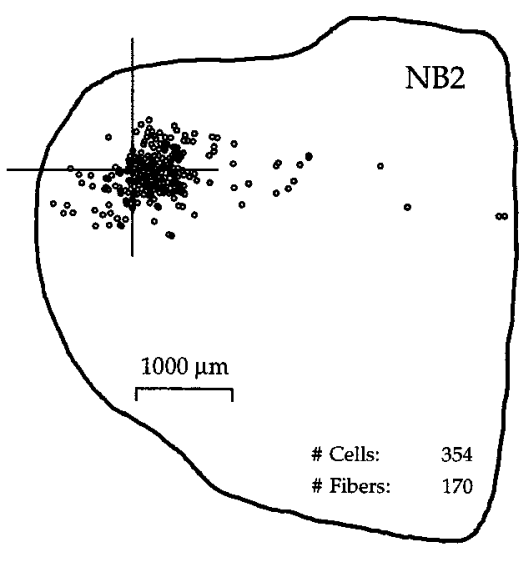

$\mathrm{D}$

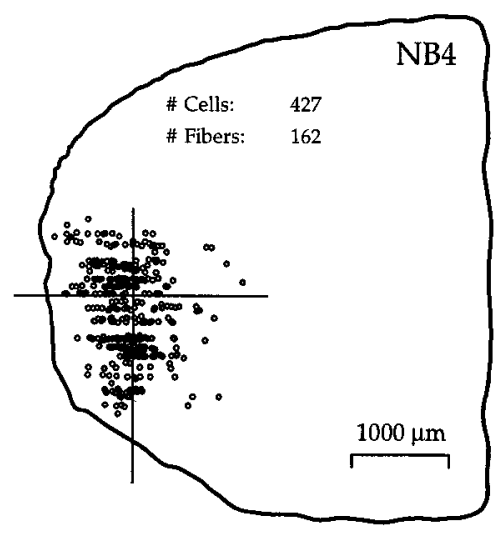

$\mathrm{G}$

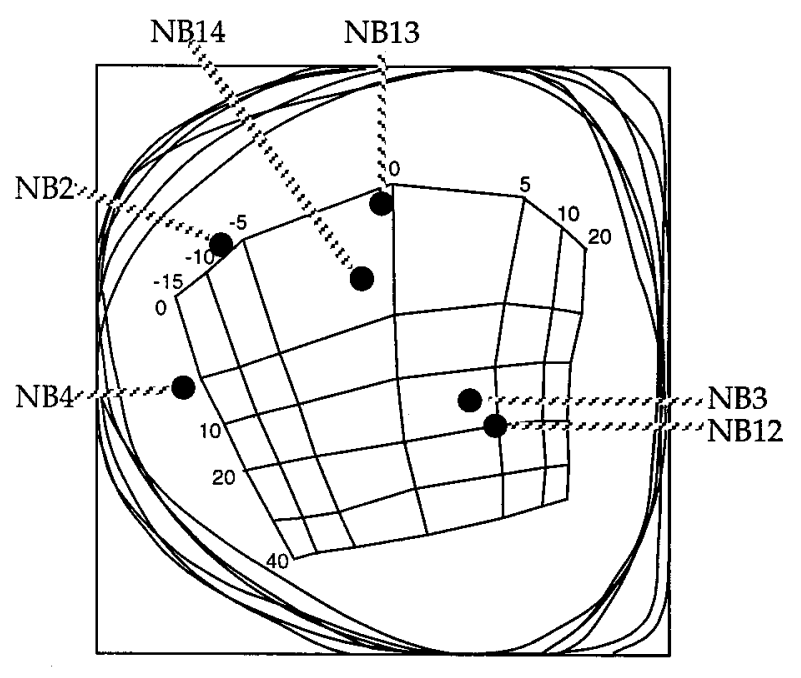

B
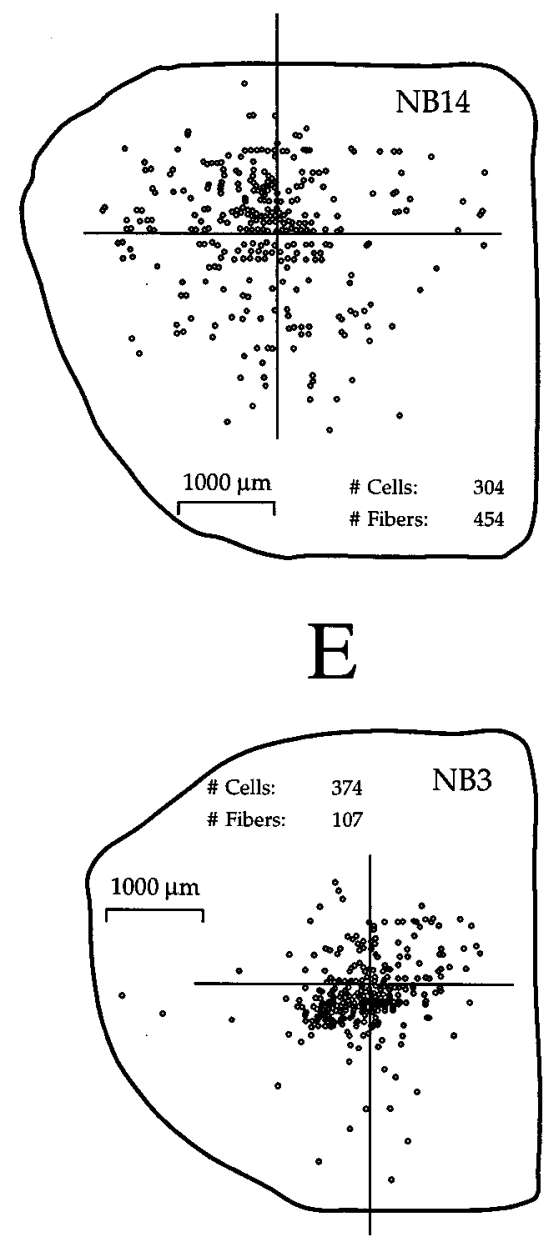

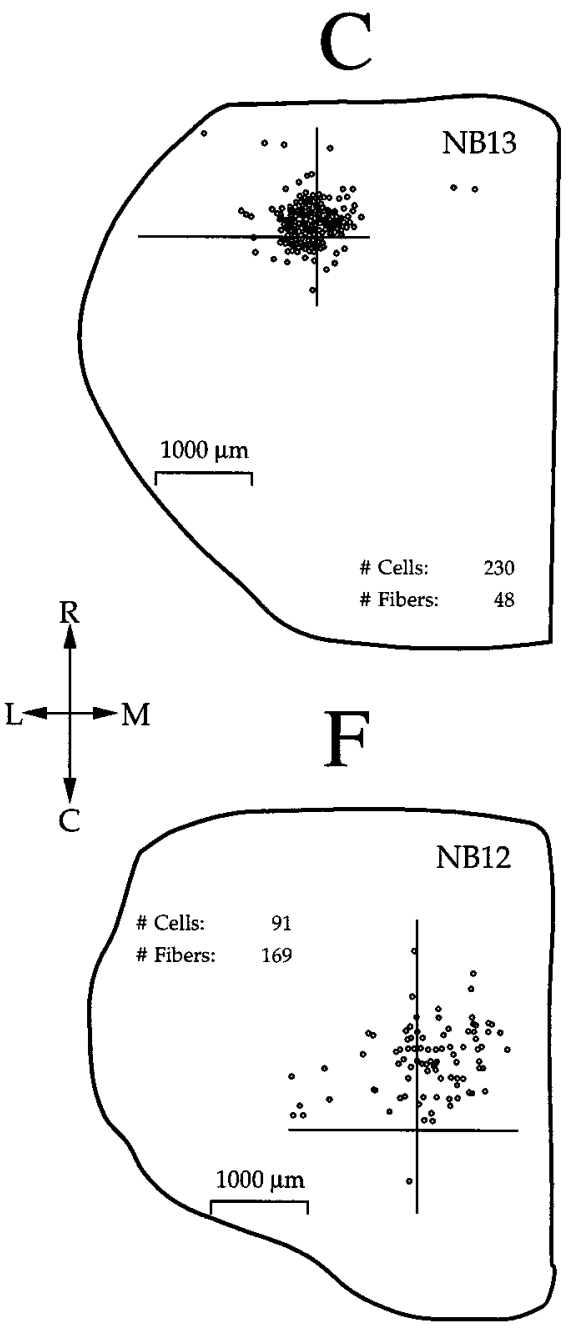

$\mathrm{H}$

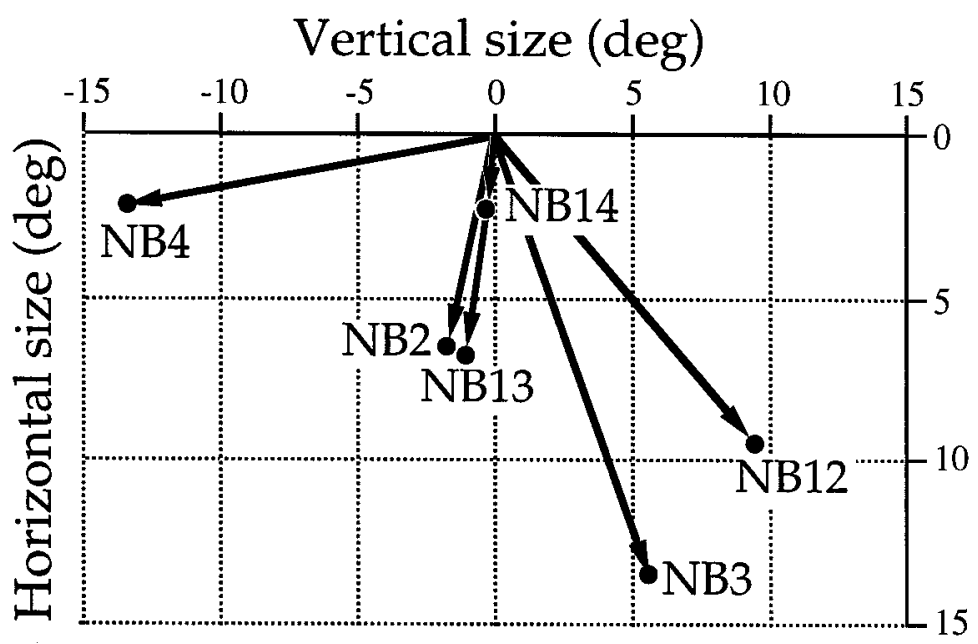

Figure 2. A-F, Horizontal plots of the outlines of the superior colliculi to illustrate the locations of stimulation and injection sites (large crosses) in different animals. Dots show the distributions of biocytin-labeled cells inside the SC. \# Cells, Number of labeled cells recovered in the SC; \# Fibers, number of labeled fibers counted at their entry in the pons; $C$, caudal; $L$, lateral; $M$, medial; $R$, rostral. $G$, Stimulation-injection sites in different animals superimposed on a retinotopic map of the SC (McIlwain, 1986) embedded within the normalized outlines of each animal's SC. Orientation arrows apply to $A-G . H$, Two-dimensional plot of the characteristic vectors (abscissa, vertical; ordinate, horizontal) of saccades evoked from corresponding collicular sites. 

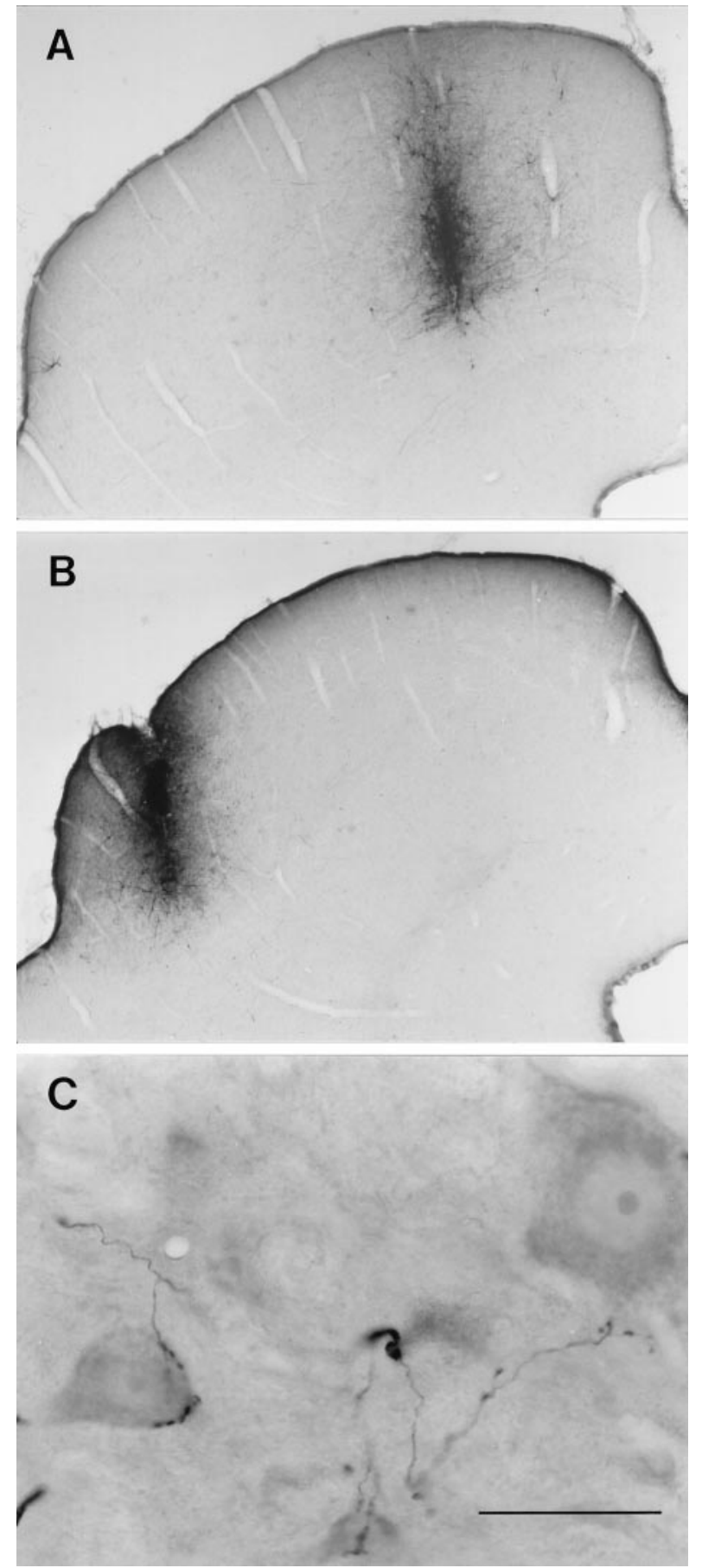

Figure 3. Photomicrographic illustration of the injection sites in NB3 $(A)$ and NB4 $(B)$ and terminal arborizations in the PPRF of NB3 $(C)$. Scale bars: $A, B, 1.25 \mathrm{~mm} ; C, 50 \mu \mathrm{m}$.

\section{RESULTS}

Figure $2 A-F$ illustrates the position of the stimulation-injection sites with respect to the outlines of the SC in the horizontal plane. The stimulated sites are distributed over a considerable portion of the mediolateral and anteroposterior extent of the SC. Their positions can be placed on the coordinates of the standard motor map of the feline SC (McIlwain, 1986) after replotting them on the normalized outlines of the SC (Fig. $2 G$ ) as described before (Grantyn et al., 1996). As shown in Figure $2 G$, the anatomical locations of the stimulation-injection sites correspond to saccade vectors with horizontal components ranging between 0 and $20^{\circ}$, and vertical components ranging between -16 (down) and $+5^{\circ}$ (up). The characteristic vectors of saccades evoked with standard stimulus parameters (see Materials and Methods) are shown in Figure $2 H$. Their horizontal components ranged from 2.1 to $13.5^{\circ}$, and their vertical components from -13.7 to $+9.2^{\circ}$. A comparison of Figure 2, $G$ and $H$, indicates only a rough correspondence between vectors effectively induced by electrical stimulation and those predicted from the anatomical locations of the stimulation sites. Such discrepancies can be partly attributed to uncertainties inherent in matching individual outlines of the SC to any standard map of the structure. Also, the SC may be anatomically heterogeneous because of local, small scale variations in the proportion of neurons representing different saccade vectors (Grantyn et al., 1996). Accordingly, we decided to study the strength of projections from labeled SC sites not in relation to the anatomically predicted, but in relation to the experimentally determined, horizontal components of saccades.

Photomicrographs in Figure 3, $A$ and $B$, illustrate the spread of the tracer in experiments NB3 and NB4, respectively. Figure 4 illustrates the tracer spread around all injection sites reconstructed from serial sections. They represent the maximum extension of the passive diffusion as determined at the level of the injection center and within $\pm 0.5 \mathrm{~mm}$ rostral and caudal to it. The subjectively defined intensity of local tissue labeling is shown by different shading. Completely opaque zones adjacent to the tracks were judged to be "strongly" labeled. "Moderate" label corresponded to zones of considerable diffuse staining that, however, allowed recognition of neuropil details. The largest, "weakly" labeled zones were delimited based on the presence of detectable diff use staining in between the fibers and cells containing the tracer injected. Within the intermediate gray layer (SGI) the diameters of weakly labeled zones measured $0.8-1.3 \mathrm{~mm}$. This provides a rough estimate of the passive tracer diffusion and of the limits of SC regions in which neuronal uptake of biocytin could occur.

In all experiments, the tips of the stimulation-injection arrays were located in the SGI. Their positions were distributed between the upper third (NB4, NB13) and the lower border (NB12, NB14) of the SGI (Fig. 4), despite the fact that we used the minimal saccade threshold to guide electrode positioning. However, the zones of passive tracer diffusion occupied the whole depth of the SGI, except for NB4 in which the lowermost edge of the layer was spared. The involvement of the optic layer (SO) was minimal in experiments NB12 and NB14. In the remaining experiments, tracer diffusion involved the SO throughout its depth. Involvement of the deep gray layer (SGP) was negligible in NB2 and NB12 and absent in the remaining four cases (Fig. 4).

Biocytin injections led to extensive labeling of cells and fibers inside the SC. Labeled cells were found in the SGI, to a lesser extent in the superficial gray layer and the SO, and occasionally in the SGP. Their number varied considerably between experiments, from 91 (NB12) to 427 (NB4). The clouds of labeled cells around the tracks are shown in Figure $2(A-F)$, projected on the horizontal plane. In some cases the center of the clouds more or less coincided with the electrode tracks, but often they did not. The majority of labeled cells were found within $\sim 1 \mathrm{~mm}$ from the injection sites. However, many remote cells were recovered as well, at distances varying between 1.4 (NB4) and $3.9 \mathrm{~mm}$ (NB2). Whereas cells neighboring the tracks may have taken up the tracer directly, through their somata or dendrites, distant cells 

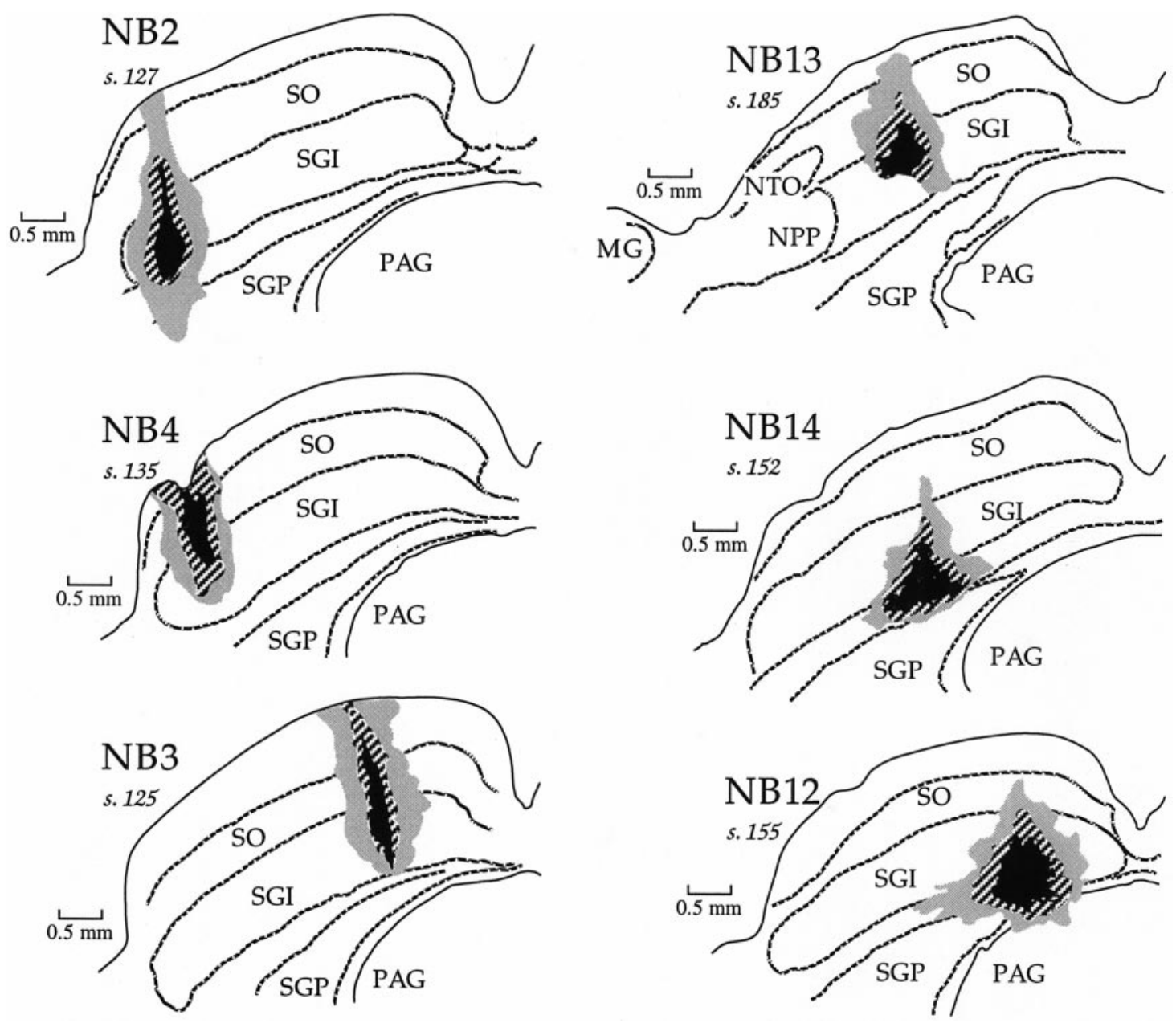

Figure 4. Camera lucida reconstructions of the stimulation-injection sites in the superior colliculus in the frontal plane. Different degrees of shading correspond to areas of intense (black), moderate (hatched), and weak (stippled) tracer deposition. Drawings show maximal extent of these areas, as seen in several (5-8) 75- $\mu$ m-thick sections centered on the electrode tracks. Note that in cases NB12 and NB14 the ventrolateral extensions of the weakly stained regions correspond to labeled fibers coursing in the intermediate white layer. These extensions were not included in the measurements of the size of diff usely labeled zones. $M G$, Medial geniculate; $N T O$, nucleus of the optic tract; $N P P$, posterior pretectal area; $S G I$, stratum griseum intermedium; $S G P$, stratum griseum profundum; $S O$, stratum opticum; $P A G$, periaqueductal gray matter.

must have been labeled retrogradely through axons damaged or interrupted by the passage of the electrode arrays. In accordance with this, we observed numerous intratectal fibers coursing in longitudinal, tangential, and oblique directions at all levels of the SC and predominantly in the SO and the SGI (Fig. 5). Because of retrograde labeling, the number and topography of cells recovered in the SC does not provide a reliable estimate of the efficacy of local biocytin uptake or of the number and position of labeled cells projecting to the burst generators. Accordingly, we did not pursue a more detailed analysis of the retrogradely labeled cells we recovered.

Two streams of labeled projection fibers could be clearly recognized in the vicinity of tracer deposits (Fig. 5). The first one consisted of radially oriented fibers that converged on the dorsolateral border of the central gray, where they entered the deep white layer and coursed toward the dorsal tegmental decussation. Inside the SC, their continuity with stained cells in the vicinity of the injection sites was often obvious without serial reconstructions. As measured in frontal sections, the size of the zones occupied by radial fibers traversing the intermediate white layer and the SGP varied from 1.0 to $1.6 \mathrm{~mm}$. These zones were somewhat larger than the extent of passive tracer diffusion estimated from the diff use staining of tissue (Fig. 5). This provides a second estimate of the size of collicular regions containing labeled cells of origin of the crossed tectoreticulospinal tract. The second stream was composed of tangential fibers coursing laterally through the intermediate white layer and, to a lesser extent, the deep gray layer. After leaving the SC at its ventrolateral border, the second stream of fibers followed the trajectory of the ipsilateral tectopontine tract through paralemniscal tegmental regions.

The PPRF is supplied by the first, crossed contingent of tectal efferent fibers. Their number was counted at their entry in the pons, $1.0-1.3 \mathrm{~mm}$ posterior to the trochlear nucleus, to evaluate the efficacy of biocytin uptake and transport specifically by fibers of this contingent. The brainstem level at which fibers were counted is illustrated in the low-power drawings of Figure 6. All predorsal bundle (PDB) fibers containing biocytin reaction product have been included, disregarding variations of staining intensity. Two examples of their distribution in the PDB are shown in Figure 6, $A_{1}$ and $B_{1}$, together with some more intensely stained collaterals visible in the same sections. In four experiments (NB3, 

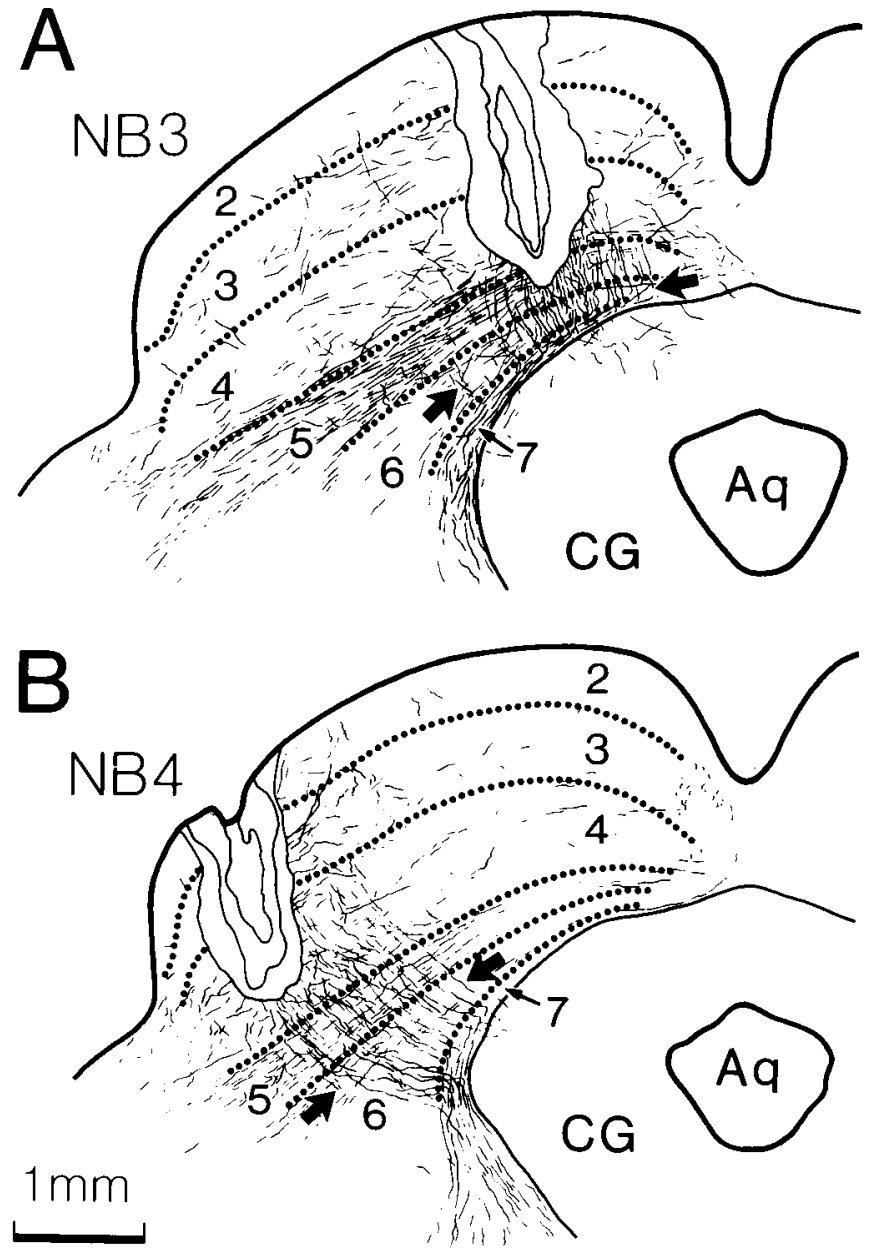

Figure 5. Distribution of labeled axons in the SC after medial ( $A, \mathrm{NB} 3)$ or lateral $(B, \mathrm{NB} 4)$ injections of biocytin. Superpositions of camera lucida tracings from five adjacent frontal sections at the level of the center of injection sites. Only axonal segments visible at low magnification $(40 \times)$ have been included. Thick arrows delimit the zone occupied by radial axons passing through the deep gray layer before joining the tectobulbospinal tract. Concentric contours correspond, from inward to outward, to dense, moderate, and weak diff use staining around the injection tracks (compare with Fig. 4). Numbers (2-7) indicate layers of the SC (superficial gray, optic, intermediate gray, intermediate white, deep gray, and deep white, respectively). $A q$, Aqueduct; $C G$, central gray.

NB4, NB12, and NB2), the number of stained PDB fibers did not differ excessively (107, 162, 169, and 170, respectively). As expected, this number was not related to the number of labeled cells in the SC. For example, the smallest (107) and the largest (170) number of axons we observed in these four experiments corresponded to 374 and 354 tectal cells, respectively. In two other experiments, we observed strong departures from this range. We found only 48 axons in experiment NB13. There were no signs of reduced tracer diffusion or uptake in this animal, as judged from the size of the tracer deposit and the number of stained collicular cells. The slightly more superficial location of the injection center in NB13 (Fig. 4) cannot explain the small number of fibers we found in the pons of this animal, because a much larger number of fibers (162) was found after a comparable injection (NB4, Fig. 4). Also, as in other experiments, fibers traversing the pons in NB13 could be followed to the bulbospinal junction. This makes unlikely an abnormally rapid fading of label with distance from the SC in NB13. Case NB14 had 454 labeled axons, thus offering the opposite extreme. In this case, the tracer deposit spread to the intermediate white layer. However, this cannot be the reason for the excessive number of PDB fibers we found in this animal. The injection sites of NB2 and NB12 also included the intermediate white layer, but we only found 170 and 169 labeled PDB axons in these experiments. Obviously, the use of the same protocol in all experiments did not eliminate all variability in labeling the PDB pathway. Therefore, bouton counts in the PPRF had to be normalized (see below).

The bouton plots of Figure 7 give overviews of terminations emanating from the two descending pathways in four of the animals we used. Each one of the overlays illustrates all the boutons we encountered in five consecutive sections through the rostral border of the nucleus reticularis pontis caudalis (NRPc; Brodal, 1957; Taber, 1961). They demonstrate three main groups of terminations: (1) in the nucleus reticularis tegmenti pontis (NRT) contralaterally, (2) in the NRPc, mainly contralaterally, and (3) in a crescent-shaped region of termination in the lateral and ventral parts of the ipsilateral reticular formation. The quantitative evaluation of pontine terminations was done on 10-15 consecutive sections from each animal. To ensure that all series of sections represented homotopic regions of the pons, the most caudal section of each of them was aligned with the caudal pole of the NRT. Because of a small difference in brainstem sizes, the center of the segments we evaluated ranged between 1.7 and $2.0 \mathrm{~mm}$ rostral to the abducens nucleus. All boutons were plotted and counted in the contralateral and ipsilateral halves of the sections. Next, separate counts were made in the region of the horizontal saccade generator. This region was defined as a rectangle extending dorsoventrally between 0 and $3 \mathrm{~mm}$ below the surface of the fourth ventricle and mediolaterally between the midline and $1.6 \mathrm{~mm}$ lateral to it (Fig. 7).

We chose these dimensions and positioned the rectangle in such a way as to obtain the best compromise between the reported locations of horizontal excitatory medium- and long-lead burst neurons in the cat. In summary, Sasaki and Shimazu (1981), who were the only ones to verify the connection of functionally identified saccadic burst neurons with the abducens nucleus $(\mathrm{AbdN})$, describe them as occupying a region extending from 0.5 to $2.0 \mathrm{~mm}$ anterior to the $\mathrm{AbdN}, 0.8$ to $1.6 \mathrm{~mm}$ from the midline, and 0.5 to $2.5 \mathrm{~mm}$ in depth. The region defined by Kaneko et al. (1981) extends further in the rostrocaudal (up to $3.5 \mathrm{~mm}$ in front of the $\mathrm{AbdN}$ ) and dorsoventral (up to 3-4 mm deep) directions and its lateral border is $1.4 \mathrm{~mm}$ from the midline. The region defined by Curthoys et al. (1981) is intermediate between the previous two in terms of rostrocaudal and dorsoventral extent, but in this case, the vast majority of burst neurons were found in strictly paramedian sites, within $0.6 \mathrm{~mm}$ from the midline. Therefore, the rectangle we selected for bouton counts includes portions of the burst neuron regions described by all three groups. As shown in Figure 7, the rectangle also contains parts of the NRT. Boutons found in this nucleus were subtracted from the total count to obtain the number of boutons in the PPRF proper.

It is instructive to describe in some detail the two extreme cases in our sample (NB4 and NB3); these differ a lot both in terms of the size of the horizontal component of the characteristic vectors of evoked saccades (Fig. 2) and in terms of the strength of their projections to the PPRF. Of the $276 \pm 36$ boutons (mean \pm SD) that were seen in each one of the midpontine sections of NB3, $150 \pm 64$ were deployed in the contralateral PPRF (range, 55255), $7 \pm 2$ in the ipsilateral PPRF, and $33 \pm 6$ in the contralateral NRT, whereas the remaining participated in the crescent-shaped 

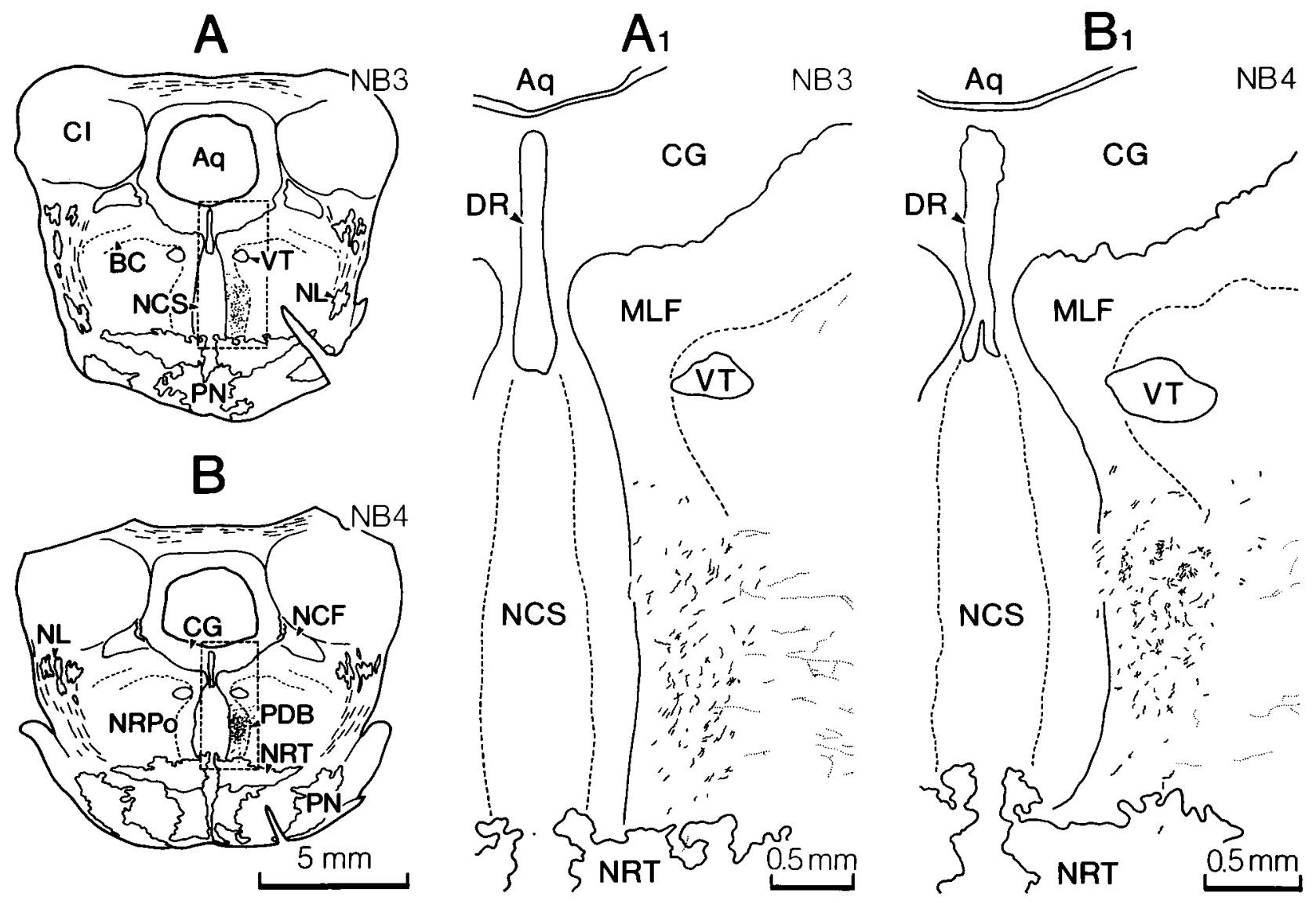

Figure 6. Camera lucida drawings of labeled predorsal bundle fibers at their entry in the pons. $A, B$, Topography of sections used to obtain the number of axons for the normalization of bouton counts in experiments NB3 and NB4, respectively. The sections are $1125 \mu \mathrm{m}(A)$ and $1275 \mu \mathrm{m}(B)$ posterior to the caudal pole of the trochlear nucleus. $A_{1}, B_{1}$. Higher power drawings of the predorsal bundle region delimited by dashed lines in $A$ and $B$, respectively. Short solid segments, Labeled descending axons. Dotted lines, Axon collaterals visible at a magnification of $100 \times$. Axon counts are $107\left(\mathrm{~A}_{1}\right)$ and $162\left(\mathrm{~B}_{1}\right) . A q$, Aqueduct; $B C$, brachium conjuctivum; $C G$, central gray; $C I$, inferior colliculus; $D R$, dorsal nucleus of the raphe; $M L F$, medial longitudinal fasciculus; $N C F$, nucleus cuneiformis; $N C S$, nucleus centralis superior; $N L$, nuclei of the lateral lemniscus; $N R P o$, nucleus reticularis pontis oralis; NRT, nucleus reticularis tegmenti pontis; $P D B$, predorsal bundle; $P N$, pontine nuclei; $V T$, ventral tegmental nucleus (of Gudden).

region of termination in the lateral part of the ipsilateral reticular formation. Many of the boutons that were recovered in the ipsilateral reticular formation were deployed by fibers that originated in the contralateral predorsal bundle and recrossed the midline. Recrossing of fibers descending in the lateral tectopontine tract has also been occasionally observed. Further caudally, a considerable number of boutons were found in the nucleus reticularis gigantocellularis, bilaterally, and the abducens nucleus, contralaterally. Much smaller bouton numbers were observed in each one of the pontine sections of NB4. They were $13 \pm 9$ (range, 2-30) in the contralateral PPRF, $16 \pm 4$ in the ipsilateral PPRF, and $5 \pm 2$ in the contralateral NRT. This difference was not caused by a weaker labeling of the predorsal bundle component. On the contrary, 162 labeled axons were counted in NB4 as they entered the pontine tegmentum $\sim 1.2 \mathrm{~mm}$ behind the trochlear nucleus, and only 107 in NB3. At the level of sections used for bouton counts, $2.5 \mathrm{~mm}$ further caudally, the number of labeled predorsal bundle fibers in NB4 was decreased by only $8 \%$, whereas a loss of $52 \%$ was observed in NB3. The rapid loss of predorsal bundle axons in NB3 appeared to correlate with an intense collateralization, whereas rostral pontine collaterals were quite rare in NB4. Thus, the bouton density of NB3 and NB4 was antipodal to the number of projecting axons but fit well the different sizes of the horizontal components of saccades evoked from collicular regions that give rise to these projections.

To examine quantitatively whether this relationship applied to all animals in our sample, and to compensate for differences between animals in terms of the number of stained axons we saw in the pons (see above), we normalized the number of boutons in the PPRF. To this end, we divided the number of boutons counted in each section through the PPRF of each animal by the number of fibers entering the pons and then multiplied by 100 to obtain the number of boutons per 100 fibers per section. Figure 8 is a scatter plot of the normalized bouton counts in the PPRF of all sections from all animals studied. These have been arranged in order of the size of the horizontal components of the "characteristic" vectors evoked in the same animals. As shown, there is a concomitant trend for the normalized number of boutons found in the contralateral PPRF to increase from $7.84 \pm 5.7$ (mean \pm SD) per 100 fibers per section in NB4 to $140.3 \pm 59.5$ (mean \pm SD) in NB3. The average normalized number of PPRF boutons are plotted against the size of the horizontal component of the "characteristic" vector of the saccades evoked from the same collicular microzone in the inset of Figure 8. The linear regression line through this data passes close to the origin, and its slope is equal to $\sim 10$ contralateral PPRF boutons per degree of hori- 

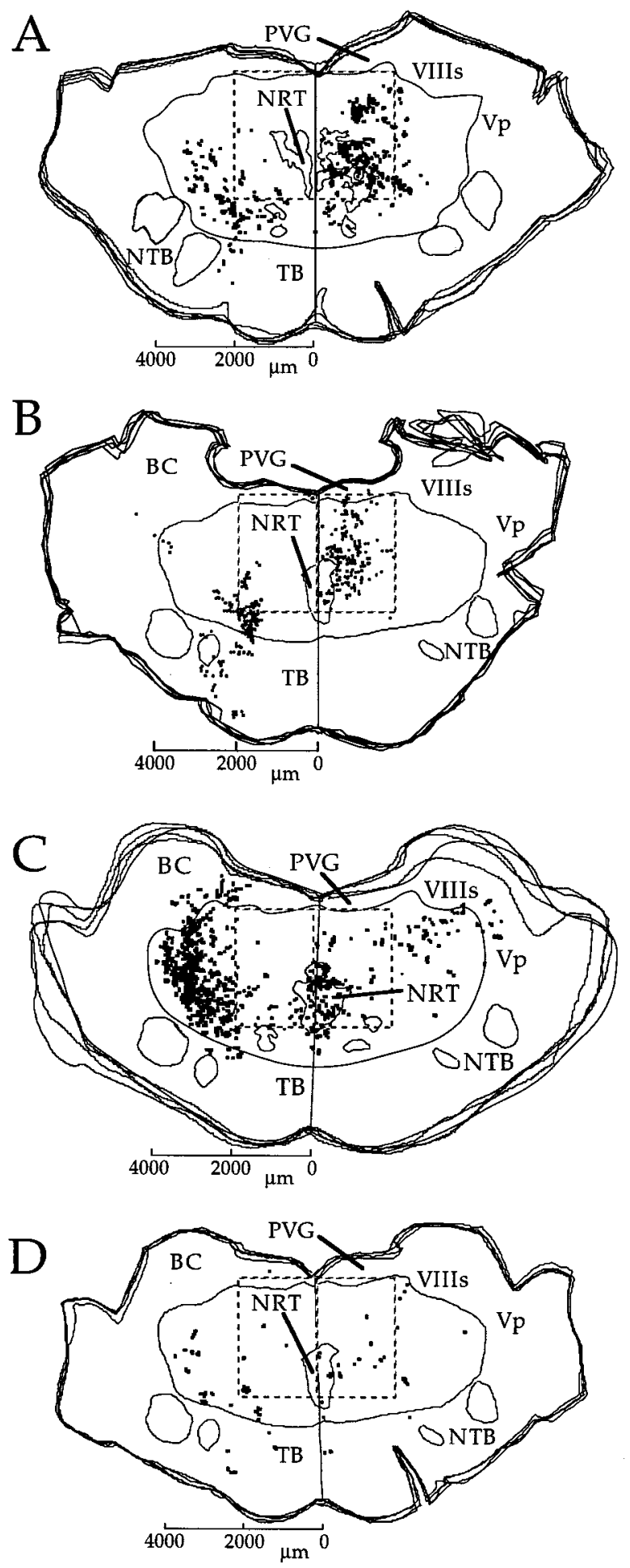

Figure 7. Composite plots (5 sections each) of biocytin-labeled boutons in coronal sections through the PPRF of NB3 $(A), \mathrm{NB} 12(B), \mathrm{NB} 2(C)$, and NB4 $(D)$. Dashed lines indicate the areas selected for bouton counts. Thin solid lines delimit the reticular core and other pontine nuclei. $V p$, Principal sensory nucleus of the trigeminal nerve; VIIIs, superior vestibular nucleus; $B C$, brachium conjuctivum; $N R T$, nucleus reticularis tegmenti pontis; $N T B$, nucleus of the trapezoid body; $P V G$, periventricular gray; $T B$, trapezoid body. zontal eye displacement per 100 fibers per section. The high correlation coefficient $(r=0.92)$ indicates that about $85 \%$ of the variance of the dependent variable (number of boutons deployed in the contralateral PPRF) can be accounted for by the independent variable (size of the horizontal component of the "characteristic" vector of saccades). ANOVA indicated that this relationship is highly statistically significant $(p<0.0001)$.

It might be argued that instead of horizontal saccade size, the normalized number of PPRF boutons is correlated with a different saccade metric such as radial size or direction. To test these hypotheses, we plotted the normalized bouton counts against the radial size and the direction of the characteristic vector of evoked saccades. The linear regression lines through data plotted in this manner were more or less flat and did not reach statistical significance (radial size: slope $=2.32$ boutons per section per 100 fibers per degree, $r=0.25, p>0.1$; direction: slope $=0.75$ boutons per section per 100 fibers per degree, $r=0.48, p>0.1$ ). We also examined the relationship between the normalized bouton counts in the PPRF and the anatomical coordinates of the injection sites in the SC. The latter were defined in two ways: (1) in terms of their relative distance from the rostral pole of the SC [measured from the caudal edge of the posterior commissure as proposed by Kanaseki and Sprague (1974) and Edwards (1977)], and (2) in terms of the size of the horizontal components of the saccade vectors that should be evoked from them according to McIlwain's (1986) sensorimotor map of the SC after its superposition on standardized outlines of the SC reconstructed from individual experiments. No significant correlations were obtained with either of these two purely anatomical parameters (relative distance from rostral pole: $r=0.05, p>0.1$; size predicted from standard map: $r=0.35, p>0.1$ ).

\section{DISCUSSION}

In this study we tested the hypothesis that the strength of anatomical projections from different regions of the SC to the PPRF covaries with the size of the horizontal components of saccades encoded by these regions. The anatomical strength of connections could depend on the density of functionally similar efferent neurons and/or the number of terminals they deploy in relevant target areas. The former was favored by Edwards and Henkel (1978) but, as pointed out in the introductory remarks, further supportive evidence has not been forthcoming. Here, we investigated the second of the two factors, the number of terminals deployed by tectal axons in the PPRF.

To our knowledge, differences between paramedian pontine projections of discrete SC regions were evaluated in only one previous study that used an anterograde degeneration technique (Kawamura et al., 1974). Within the resolution limit of their method, the authors concluded that there are no quantitative differences between different parts of the SC in terms of the density of their projections. Subsequent studies of collicular efferent projections used the anterograde autoradiographic tracing technique in cats (Edwards and Henkel, 1978; Huerta and Harting, 1982; Cowie and Holstege, 1992) and monkeys (Harting, 1977). Relationships between the location of injection sites and the density of terminations were not examined in these studies.

The biocytin injections we used spanned a substantial fraction of the rostrocaudal extent of the SC. In all experiments, the tips of injection pipettes were located in the SGI so that variation in the depth of labeled regions was small compared with the extent of the dendritic fields $(600-1600 \mu \mathrm{m})$ of the cells of origin of the predorsal bundle (Moschovakis and Karabelas, 1985). From the 


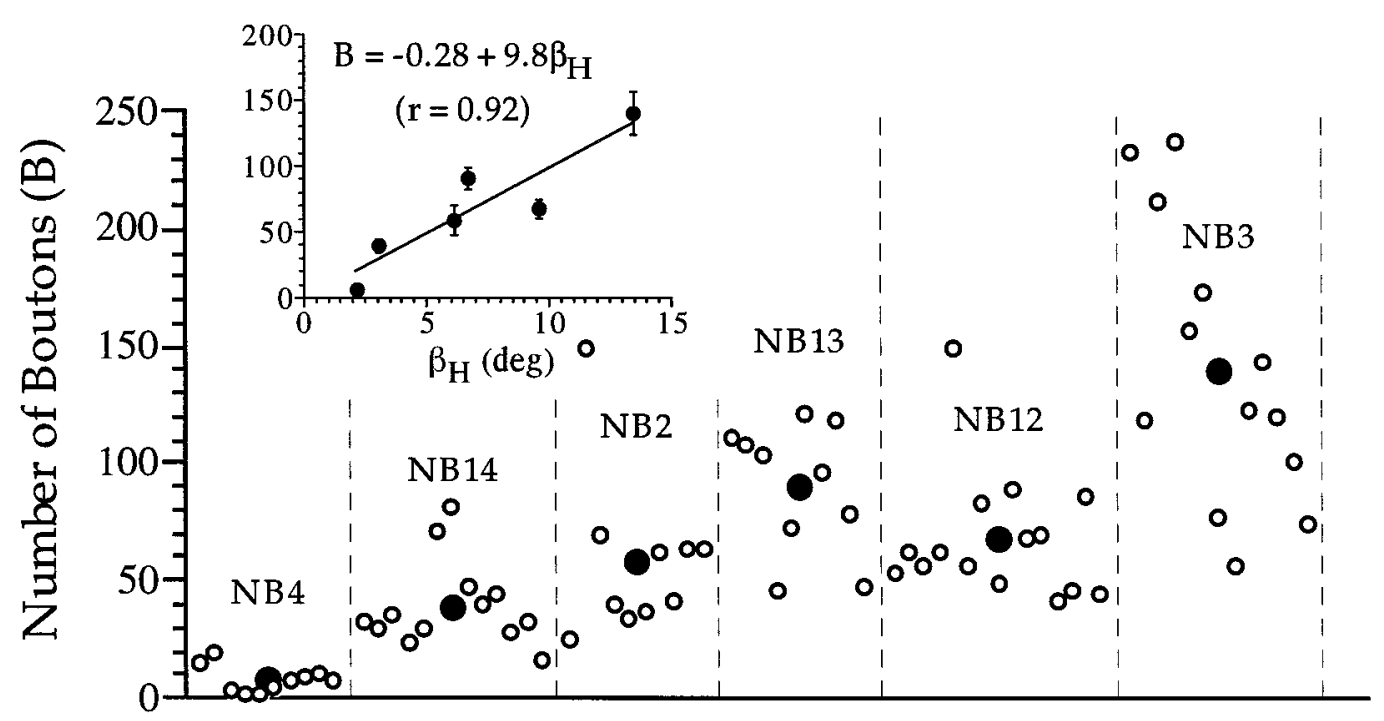

Figure 8. Scatter plot of the number of boutons per 100 fibers (ordinate) deployed in the PPRF. Dashed vertical lines separate sections that belong to different animals. Small open circles indicate the number of boutons observed in adjacent individual $75 \mu \mathrm{m}$ sections, whereas large solid circles indicate the average for the animal indicated. The inset is a plot of the average number of boutons deployed in the PPRF per 100 fibers per section ( $B$; ordinate) from each one of the injection sites versus the size of the horizontal component of the characteristic vector of the saccades evoked from the same site $\left(\beta_{\mathrm{H}}\right.$; abscissa). Error bars indicate the SEM. The solid line is the linear regression line through the data and obeys the equation displayed.

duration of the pulses we used and previous data on currentdistance relationships (for review, see Tehovnik, 1996), we estimate that activated cell bodies or axons are located within $0.4-$ $0.55 \mathrm{~mm}$ from the electrode tip for current intensities equal to 20 $\mu \mathrm{A}$ and within $0.55-0.85 \mathrm{~mm}$ for current intensities equal to 50 $\mu \mathrm{A}$. These estimates match well the distances of tracer diffusion $(0.5-0.7 \mathrm{~mm})$ as well as the size of the zones occupied by radially oriented labeled axons traversing the SGI to join the tectobulbospinal tract. Therefore, it is safe to conclude that the cells that were directly labeled with biocytin correspond reasonably well to the cells that were directly excited by current.

On the other hand, the number of labeled PDB axons varied almost by 10 -fold despite the fact that the volume of tracer injected and the histochemical processing of the tissue were the same in all experiments. Accordingly, we had to normalize bouton counts by calculating the number of boutons in the PPRF per 100 labeled fibers. Our study demonstrates that the normalized number of boutons deployed in the PPRF by different collicular microzones increases at a rate of $\sim 10$ per section per 100 fibers per degree of horizontal size of the characteristic vector encoded by the same zones. It is important to note that this correlation applies to the characteristic vectors of saccades actually evoked from the labeled sites and not to the anatomical coordinates of the injection sites as they relate to standard motor maps. This is consistent with the fact that evoked saccade vectors cannot be predicted with sufficient precision from the coordinates of the stimulation sites on a standard motor map matched to the outlines of the SC. For example, horizontal components of saccades evoked from the isoazimuth region of $5-10^{\circ}$ of the cat range between 6 and $13^{\circ}$ (McIlwain, 1990). The situation is similar in the monkey. Stimulation points along the $10^{\circ}$ isoamplitude curve of this species give rise to saccades that range from $4.5-14^{\circ}$ (Robinson, 1972). Other parameters that might be related to the strength of pontine SC projections include saccade direction and radial size. Neither proved statistically significant. We conclude that bouton counts in the PPRF increase in proportion to only one specific parameter, the horizontal amplitude of the charac- teristic vector encoded by the activated population of tectal efferent neurons.

The PPRF contains long-lead and medium-lead burst neurons discharging in relation to horizontal saccades and is, thus, thought to encompass the "horizontal saccadic burst generator" (for review, see Hepp et al., 1989; Moschovakis et al., 1996). In the cat, the locations of MLBs and LLBs have been mapped in three studies (Curthoys et al., 1981; Kaneko et al., 1981; Sasaki and Shimazu, 1981) that are not in perfect agreement. As pointed out in the Results section, we chose for bouton counts a PPRF region that contains LLBs and MLBs according to all three studies. The region selected is in the rostral part of the nucleus reticularis pontis caudalis and corresponds to an area that receives a dense tectal projection as revealed by anterograde degeneration and autoradiographic tracing (Kawamura et al., 1974; Harting, 1977; Huerta and Harting, 1982; Cowie and Holstege, 1992). Terminations in the same area have also been demonstrated by intracellular staining of "visuomotor" tectal projection neurons (Olivier et al., 1993) and tectal long-lead burst neurons (TLLBs; Scudder et al., 1996). These anatomical projections do engage the saccadic burst generator, as shown by the monosynaptic excitatory responses of primate LLBs (Raybourn and Keller, 1977) and feline MLBs (Hikosaka and Kawakami, 1977; Chimoto et al., 1996) to SC stimulation.

The region of the feline saccadic burst generator in the PPRF also contains reticulospinal neurons that generate phasic or phasic-decremental discharges coupled to horizontal saccades and head movements (Grantyn and Berthoz, 1987; Isa and Naito, 1995). Several studies suggest that the gaze-related activity of reticulospinal neurons is derived from tectoreticulospinal neurons (TRSNs) that make collateral connections with the PPRF (Grantyn and Berthoz, 1985; Olivier et al., 1993). In agreement with this, TRSN burst activity is related to both saccades and head acceleration in head-free cats (Munoz et al., 1991). Therefore, the terminations of TRSNs in the PPRF could contact reticulospinal neurons controlling head movements and eye-head synergies in addition to cells of the saccadic burst generator 
(LLBs and MLBs). Distinguishing between boutons that contact one or the other class of premotor neurons does not seem necessary because eye and head movements in the cat are strongly coupled during combined gaze shifts (Guitton et al., 1984).

Focal injections of biocytin in the SC would also label PDB axons whose activity is unrelated to saccades, and more generally to orienting movements (Grantyn and Berthoz, 1985; Moschovakis et al., 1988; Istvan et al., 1994). Similarly, the medial pontine reticular formation, including the region we have studied, contains many cells whose activity is not overtly related to gaze shifts (Siegel, 1979; Siegel and Tomaszcewski, 1983; Isa and Naito, 1995; Kitama et al., 1995). By definition, cells unrelated to orienting behavior are not particular as to the direction and the amplitude of orienting movements. Their presence, both in the population of labeled SC neurons and among their target cells in the PPRF, should not, therefore, influence the observed saccaderelated gradient of bouton counts.

To implement the spatiotemporal transformation, several models of the saccadic system have assumed a differential weighing of synaptic connections so that TLLBs that discharge before larger saccades exert a stronger excitatory influence on LLBs and MLBs than TLLBs that discharge before smaller saccades (for review, see Moschovakis and Highstein, 1994; Moschovakis et al., 1996). To illustrate how stronger connections could be transformed to MLB bursts of longer duration, let us consider the information flow through eye displacement models of the burst generator (Jürgens et al., 1981; Scudder, 1988; Moschovakis, 1994). Computer simulations of these models produce LLB bursts that can be thought of as low-pass filtered versions of the bursts emitted by collicular neurons (TLLBs). The resettable integrator cells supposedly receive excitatory input from LLBs (Moschovakis, 1994) or MLBs (Scudder, 1988) and suppress the discharge of LLBs. When their augmenting discharge surpasses the discharge of LLBs, the latter stop discharging. This moment is delayed if the TLLB excitatory drive on LLBs is made stronger, for example, when the activated TLLBs are the ones discharging for bigger saccades and deploying stronger projections. Such a hypothetical circuit suffices to ensure the proportionality of three variables: (1) the strength of projections of topographically delimited populations of active TLLBs, (2) the duration of MLB bursts, and (3) the duration of saccades. However, its correct performance depends on arbitrary choices of feedback and feedforward connection strengths and integrator time constant. The present study is the first to demonstrate that one of its assumptions, namely the differential weighing of SC projections to the saccadic burst generator, is realistic from the point of view of anatomy. The verisimilitude of other features remains to be experimentally determined.

\section{REFERENCES}

Adams JC (1981) Heavy metal intensification of DAB-based HRP reaction product. J Histochem Cytochem 29:775.

Brodal A (1957) The reticular formation of the brain stem. Anatomical aspects and functional correlations. Edinburgh: Oliver and Boyd.

Chimoto S, Iwamoto Y, Shimazu H, Yoshida K (1996) Monosynaptic activation of medium-lead burst neurons from the superior colliculus in the alert cat. J Neurophysiol 75:2658-2661.

Cowie RJ, Holstege G (1992) Dorsal mesencephalic projections to pons, medulla, and spinal cord in the cat: limbic and non-limbic components. J Comp Neurol 319:536-559.

Curthoys IS, Nakao S, Markham CH (1981) Cat medial pontine reticular neurons related to vestibular nystagmus: firing pattern, location and projection. Brain Res 222:75-94.

Edwards SB (1977) The commissural projection of the superior colliculus in the cat. J Comp Neurol 173:23-40.
Edwards SB, Henkel CK (1978) Superior colliculus connections with the extraocular motor nuclei in the cat. J Comp Neurol 179:451-468.

Grantyn A, Berthoz A (1985) Burst activity of identified tectoreticulospinal neurons in the alert cat. Exp Brain Res 57:417-421.

Grantyn A, Berthoz A (1987) Reticulospinal neurons participating in the control of synergic eye and head movements during orienting in the cat. I. Behavioral properties. Exp Brain Res 66:339-354.

Grantyn AA, Dalezios Y, Kitama T, Moschovakis AK (1996) Neuronal mechanisms of two-dimensional orienting movements in the cat. I. A quantitative study of saccades and slow drifts produced in response to the electrical stimulation of the superior colliculus. Brain Res Bull 41:65-82.

Grantyn AA, Dalezios Y, Kitama T, Moschovakis AK (1997) An anatomical basis for the spatio-temporal transformation in the saccadic system. Soc Neurosci Abstr 23:1295.

Guitton D, Crommelinck M, Roucoux A (1980) Stimulation of the superior colliculus in the alert cat. I. Eye movements and neck EMG activity evoked when the head is restrained. Exp Brain Res 39:63-73.

Guitton D, Douglas RM, Volle M (1984) Eye-head coordination in cats. J Neurophysiol 52:1030-1050.

Harting JK (1977) Descending pathways from the superior colliculus: an autoradiographic analysis in the rhesus monkey (Macaca mulatta). J Comp Neurol 173:583-612.

Hepp K, Henn V, Vilis T, Cohen B (1989) Brainstem regions related to saccade generation. In: The neurobiology of saccadic eye movements (Wurtz RE, Goldberg ME, eds), pp 105-212. Amsterdam: Elsevier.

Hikosaka O, Kawakami T (1977) Inhibitory reticular neurons related to the quick phase of vestibular nystagmus: their location and projection. Exp Brain Res 27:377-396.

Horikawa K, Armstrong WE (1988) A versatile means of intracellular labeling: injection of biocytin and its detection with avidin conjugates. J Neurosci Methods 25:1-11.

Huerta MF, Harting JK (1982) Tectal control of spinal cord activity: neuroanatomical demonstration of pathways connecting the superior colliculus with the cervical spinal cord grey. In: Descending pathways to the spinal cord (Kuypers HGJM, Martin GF, eds), pp 293-328. Amsterdam: Elsevier.

Isa T, Naito K (1995) Activity of neurons in the medial pontomedullary reticular formation during orienting movements in alert head-free cats. J Neurophysiol 74:73-95.

Istvan PJ, Dorris MC, Munoz DP (1994) Functional identification of neurons in the monkey superior colliculus that project to the paramedian pontine reticular formation. Soc Neurosci Abstr 20:141.

Jürgens R, Becker W, Kornhuber HH (1981) Natural and drug-induced variations of velocity and duration of human saccadic eye movements: evidence for a control of the neural pulse generator by local feedback. Biol Cybern 39:87-96.

Kanaseki T, Sprague JM (1974) Anatomical organization of pretectal nuclei and tectal laminae in the cat. J Comp Neurol 158:319-338.

Kaneko CRS, Evinger C, Fuchs AF (1981) Role of cat pontine burst neurons in generation of saccadic eye movements. J Neurophysiol 46:387-408.

Kawamura K, Hashikawa T (1978) Cell bodies of origin of reticular projections from the superior colliculus in the cat: an experimental study with the use of horseradish peroxidase as a tracer. J Comp Neurol 182:1-16.

Kawamura K, Brodal A, Hoddevik GH (1974) The projection of the superior colliculus onto the reticular formation of the brain stem: an experimental anatomical study in the cat. Exp Brain Res 19:1-19.

Keller E (1980) Oculomotor specificity within subdivisions of the brain stem reticular formation. In: The reticular formation revisited (Hobson JA, Brazier MAB, eds), pp 227-240. New York: Raven.

Kitama T, Grantyn A, Berthoz A (1995) Orienting related eye-neck neurons of the medial ponto-bulbar reticular formation do not participate in horizontal canal-dependent vestibular reflexes of alert cats. Brain Res Bull 38:337-347.

McIlwain JT (1982) Lateral spread of neural excitation during microstimulation in intermediate gray layer of cat's superior colliculus. J Neurophysiol 47:167-178.

McIlwain JT (1986) Effects of eye position on saccades evoked electrically from the superior colliculus of alert cats. J Neurophysiol 55:97-112.

McIlwain JT (1988) Effects of eye position on electrically evoked saccades: a theoretical note. Vis Neurosci 1:239-244.

McIlwain JT (1990) Topography of eye-position sensitivity of saccades 
evoked electrically from the cat's superior colliculus. Vis Neurosci 4:289-298.

Moschovakis AK (1994) Neural network simulations of the primate oculomotor system. I. The vertical saccadic burst generator. Biol Cybern 70:291-302.

Moschovakis AK, Highstein SM (1994) The anatomy and physiology of primate neurons that control rapid eye movements. Annu Rev Neurosci $17: 465-488$.

Moschovakis AK, Karabelas AB (1985) Observations on the somatodendritic morphology and axonal trajectory of intracellularly HRPlabeled efferent neurons located in the deeper layers of the superior colliculus of the cat. J Comp Neurol 239:276-308.

Moschovakis AK, Karabelas AB, Highstein SM (1988) Structurefunction relationships in the primate superior colliculus. II. Morphological identity of presaccadic neurons. J Neurophysiol 60:263-302.

Moschovakis AK, Scudder CA, Highstein SM (1996) The microscopic anatomy and physiology of the mammalian saccadic system. Prog Neurobiol 50:133-254.

Munoz DP, Guitton D, Pelisson D (1991) Control of orienting gaze shifts by the tectoreticulospinal system in the head-free cat. III. Spatiotemporal characteristics of phasic motor discharges. J Neurophysiol 66:1642-1666.

Olivier E, Chat M, Grantyn A (1991) Rostrocaudal and lateromedial density distributions of superior colliculus neurons projecting in the predorsal bundle and to the spinal cord: a retrograde HRP study in the cat. Exp Brain Res 87:268-282.

Olivier E, Grantyn A, Chat M, Berthoz A (1993) The control of slow orienting movements by tecto-reticulo-spinal neurons in the cat: behaviour, discharge patterns and underlying connections. Exp Brain Res 93:435-449.

Paré M, Crommelinck M, Guitton D (1994) Gaze shifts evoked by stimulation of the superior colliculus in the head-free cat conform to the motor map but also depend on stimulus strength and fixation activity. Exp Brain Res 101:123-139.
Raybourn MS, Keller EL (1977) Colliculoreticular organization in primate oculomotor system. J Neurophysiol 40:861-878.

Robinson DA (1972) Eye movements evoked by collicular stimulation in the alert monkey. Vision Res 12:1795-1808.

Sasaki S, Shimazu H (1981) Reticulovestibular organization participating in generation of horizontal fast eye movement. Ann NY Acad Sci 374:130-143.

Scudder CA (1988) A new local feedback model of the saccadic burst generator. J Neurophysiol 59:1455-1475.

Scudder CA, Moschovakis AK, Karabelas AB, Highstein SM (1996) Anatomy and physiology of saccadic long-lead burst neurons recorded in the alert squirrel monkey. I. Descending projections from the mesencephalon. J Neurophysiol 76:332-352.

Siegel JM (1979) Behavioral functions of the reticular formation. Brain Res Rev 1:69-105.

Siegel JM, Tomaszcewski KS (1983) Behavioral organization of reticular formation: studies in the unrestrained cat. I. Cells related to axial, limb, eye and other movements. J Neurophysiol 50:696-716.

Sparks DL, Mays LE (1980) Movement fields of saccade-related burst neurons in the monkey superior colliculus. Brain Res 190:39-50.

Stanton GB, Greene RW (1981) Brain stem afferents to the periabducens reticular formation (PARF) in the cat. An HRP study. Exp Brain Res 44:419-426.

Stein BE, Goldberg SJ, Clamann HP (1976) The control of eye movements by the superior colliculus in the alert cat. Brain Res 118:469-474.

Straschill M, Rieger P (1973) Eye movements evoked by focal stimulation of the cat's superior colliculus. Brain Res 59:211-227.

Taber E (1961) The cytoarchitecture of the brain stem of the cat. J Comp Neurol 116:27-69.

Tehovnik EJ (1996) Electrical stimulation of neural tissue to evoke behavioral responses. J Neurosci Methods 65:1-17.

Wurtz RE, Goldberg ME (1972) Activity of superior colliculus in behaving monkey. III. Cells discharging before eye movements. J Neurophysiol 35:575-586. 